\title{
Left Ventricular Papillary Fibroelastoma
}

\author{
Renato Jorge Alves, Alexandre H. Borin, Carlos Renato De Oliveira, Raphael Puig, Ana Carolina Mercê, Kenji Nakiri \\ Hospital Santa Cruz, São Paulo, SP - Brazil
}

We evaluated the case of a patient with Papillary Fibroelastoma (PFE) that presented embolization to the upper right limb. The patient was submitted to percutaneous embolectomy, with fragment removal. The diagnosis was confirmed by transthoracic echocardiogram and anatomopathological analysis of the fragment. The patient chose to undergo the conservative clinical treatment and the follow-up has shown good evolution with no disease recurrence to date.

We decided to use this rare and interesting case with the objective of reviewing the current literature and discuss the best therapeutic management.

\section{Introduction}

The primary heart tumors are rarer than metastatic ones, with an incidence of $0.002 \%-0.3 \%$. Approximately $75 \%$ of the tumors are benign and, of these, 50\% of myxomas ${ }^{1}$. The Papillary Fibroelastoma (PFE) is a tumor that is rarely diagnosed during life and corresponds to approximately $8 \%$ of heart tumors ${ }^{2}$.

\section{Case report}

We report on a 71-year-old female patient with dyslipidemia, arterial hypertension and obesity. The patient came to our Service on February 24, 2007, complaining of pain in the upper right limb that had lasted for one hour. The pain had started in the hand and progressed up to the arm and shoulder, associated with a decrease in motor strength and sensitivity. The high-intensity, throbbing pain at rest showed no improvement after change in position or medication and was accompanied by malaise. At the physical examination, the patient was conscious, eupneic, acyanotic, with normal skin color, anicteric and afebrile, with regular heart rhythm, normal heart sounds and no murmurs. The heart rate was 88 b.p.m.

\section{Key Words}

Neoplasms/therapy; heart; embolectomy.

\footnotetext{
Mailing address: Renato Jorge Alves •

Hospital Santa Cruz, Diretoria Médica - Rua Santa Cruz, 398 - Vila Mariana - 04122-000 - São Paulo - Brazil

E-mail: rjorge@cardiol.br, renatoalves178@gmail.com

Manuscript received December 22, 2008; revised manuscript received June 03, 2009; accepted July 27, 2009.
}

and blood pressure was 180/100 $\mathrm{mmHg}$. The upper right limb showed decreased radial pulse and signs of hypoperfusion. The electrocardiogram (ECG) showed sinus rhythm, with evidence of left atrial overload and the chest X-ray did not show any significant alterations. The surgical exploration was chosen, together with the percutaneous embolectomy of the humeral artery and fragment removal, which was sent to anatomopathological analysis. The patient presented improvement in the clinical picture and a transthoracic echocardiogram was performed at the complementary assessment, two days after the hospitalization, on February 26, 2007, which showed a small mobile, triangular-shaped mass, adhered to the apical septal region, on the left ventricular surface, measuring $8 \times 5 \mathrm{~mm}$.

When the patient was informed about the finding, she reported that during the routine echocardiogram performed in 1999, an intracavitary mass had been observed in the left ventricle, confirmed by a transesophageal echocardiogram (mid-apical pedunculated image measuring $9 \times 8 \mathrm{~mm}$ ). At the time, a surgical procedure was indicated for the resection of the mass, with a preoperative investigation that included cardiac catheterism, which did not show coronary alterations.

The patient, however, refused the surgical treatment and chose to undergo the clinical follow-up. During this follow-up, the transesophageal echocardiogram performed on December 13, 2004, showed two tumor masses adhered to the distal mid-third of the left ventricular septal wall, which were mobile, irregular, with heterogeneous aspect and measured $18 \times 12 \mathrm{~mm}$ (the larger one) and $8.9 \times 10 \mathrm{~mm}$ (the smaller one), below the mitral valve posterior leaflet. A transthoracic echocardiogram was carried out on August 23, 2005, which showed a small mass adhered to the distal mid-third of the septal wall on the left ventricular surface (Figure 1).

At this time, a chest computed tomography (CT) was requested, which was carried out on September 05, 2005. The CT showed an apical vegetation-lesion on the anteroseptal wall of the left ventricle, with irregular contours and low attenuation, measuring $22 \times 17 \times 14 \mathrm{~mm}$. The patient had refused any interventionist treatment so far (September 2005) and the symptoms had recurred only recently (February 2007). The tumor, originated from an arterial embolectomy, was formed by irregular fragments of darkish tissue and had a spongy aspect, measuring $2.0 \times 1.5 \times 0.5 \mathrm{~cm}$. At the microscopy, the histological assessment showed it was a Papillary Fibroelastoma (PFE), described below, in the four panels of Figure 2.

The diagnosis of PFE was attained based on the anatomopathological analysis. The clinical follow-up was 


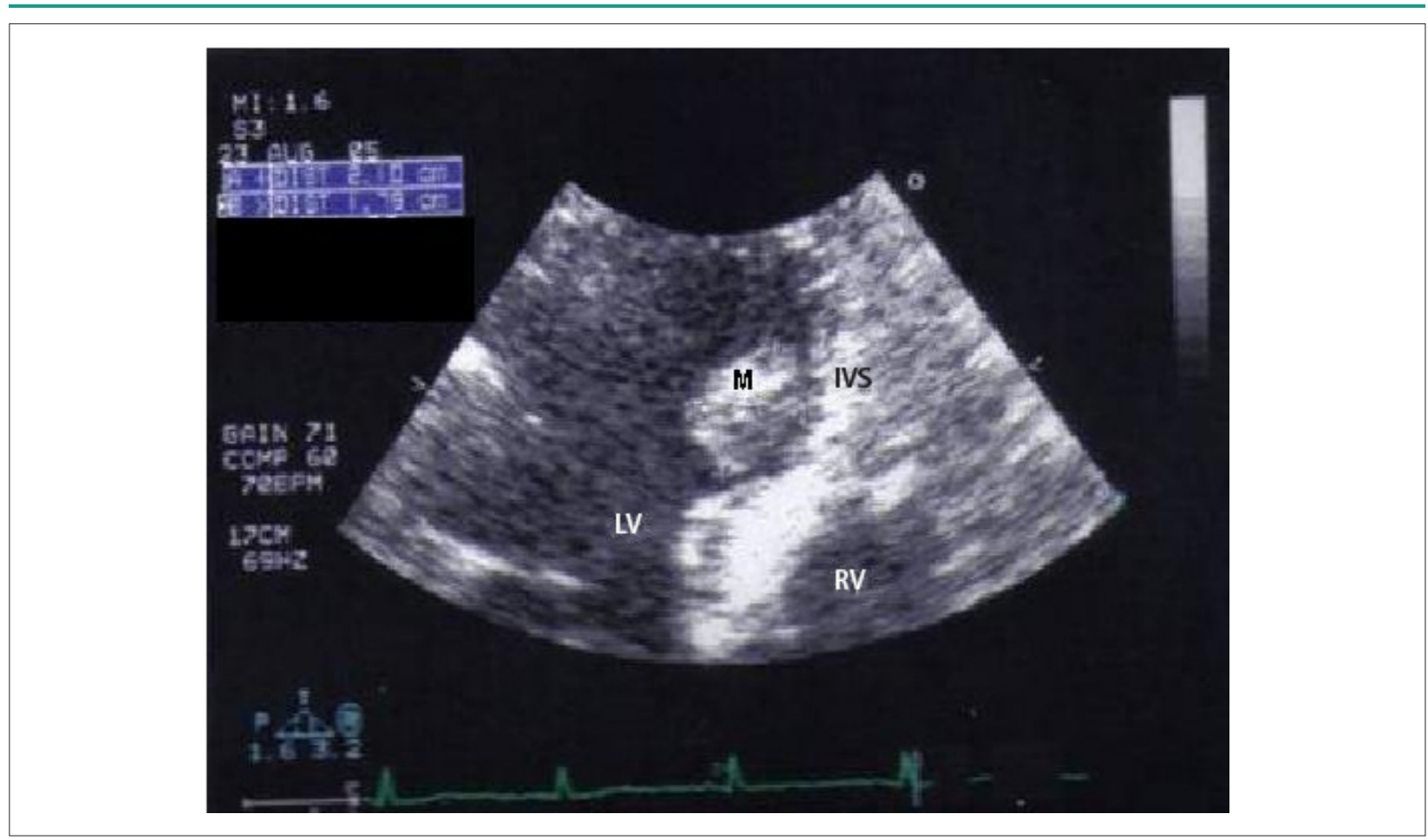

Figure 1 - Transthoracic echocardiogram: tumor mass adhered to the distal mid-third of the septal wall of the left ventricular surface. M - tumor mass; $L V$ - left ventricle; $R V$ - right ventricle; IVS - interventricular septum.

indicated, due to the tumor location (interventricular septum, with a higher chance of reaching the bundle of Hiss during the intervention), benign nature of the tumor and prognosis and the patient was kept on anticoagulation therapy. To date, the patient has presented good clinical evolution, with no recurrence of embolization.

\section{Discussion}

The advances in the noninvasive techniques of cardiovascular imaging diagnosis, especially by echocardiography, computed tomography and nuclear magnetic resonance have significantly improved the diagnostic assessment and allowed the faster identification of intracardiac masses.

The PFE is an incidental finding in many cases, although the clinical presentation is variable in symptomatic patients and depends on the tumor location, mobility and size. The PFE is a rare benign tumor, more commonly observed in the heart valves ${ }^{3}$.

The most feared complication is the systemic embolization, particularly to the brain or coronary circulation. It is not clear whether the embolus has a tumor or platelet origin and whether systemic anticoagulation can prevent such events. The most common clinical presentations described are the cerebrovascular accident (CVA) or transient ischemic attack (TIA). Other forms include: angina pectoris, myocardial infarction, sudden death, heart failure, syncope, pulmonary embolism, blindness, peripheral embolism or renal infarction ${ }^{4}$
Tumor mobility is a significant independent predictor of mortality or nonfatal embolization. The therapeutic proposal, when they are pedunculated, is the surgical resection aiming at preventing brain, pulmonary, coronary or peripheral embolic phenomena ${ }^{4}$.

The electrocardiographic findings are usually unspecific and atrial arrhythmias eventually occur. The chest X-ray can demonstrate signs of increased cardiac chambers, hypertension or pulmonary congestion, particularly if the tumor is occluding the mitral valve. However, the transthoracic echocardiogram is the ideal method for tumor diagnosis and characterization ${ }^{5}$. Usually, the transthoracic echocardiogram discloses the mass, with its varying proportions, mobile or not, pedunculated or sessile, of round, oval or irregular shape.

In a case-control study, the sensitivity and specificity of the echocardiogram were $88.9 \%$ and $87.8 \%$, respectively ${ }^{6}$. The improvement in the diagnostic power and the disseminated use of two-dimensional echocardiography resulted in the increased detection of primary cardiac tumors ${ }^{7}$.

Magnetic resonance has a significant importance in the detection and characterization of cardiac tumors and, in some cases, it can disclose, with a higher degree of precision, the size, shape and characteristics of the tumor surface, when compared to the two-dimensional echocardiography. The magnetic resonance discloses the mass in the valvular leaflet or cardiac chamber and the presence of gadolinium contrast enhancement in the tumor mass increases the degree of suspicion ${ }^{8}$.

The coronary angiography allows the visualization of total 


\section{Case Report}

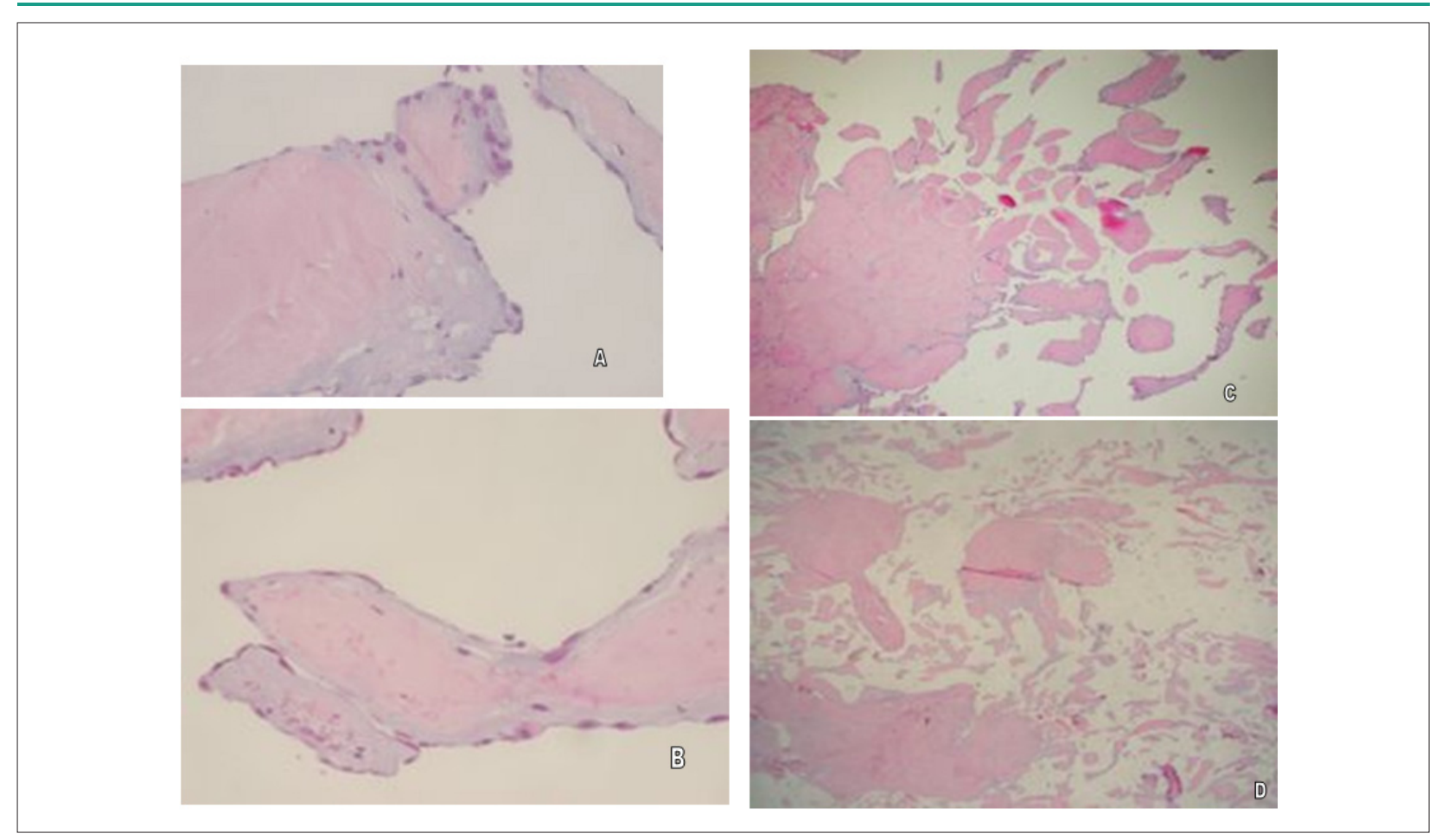

Figure 2 - Anatomopathological analysis. Papillary Fibroelastoma. Hematoxylin-eosin (HE) stain. Multiple papillary projections coated by endothelial cells, narrow outer layer of conjunctive tissue and avascular central, homogenous and hyalinized area (Panels A, B, C and D).

occlusions of the coronary arteries, as well as aneurysmatic dilatations and narrowings secondary to tumor emboli, in addition to providing data that significantly affect the surgical approach ${ }^{9}$. For symptomatic patients, the surgical resection is the treatment of choice, always attempting to preserve the valvular tissue and function.

In asymptomatic individuals, the surgical procedure is controversial, with tumor mobility being the determinant factor for the surgical indication, as it is an independent predictor of embolization and death. The surgery can be curative in most cases.

The resection of the normal tissue at the base of the pedicle implantation is important to prevent recurrence. The defect created can be sutured directly or corrected using a bovine pericardial patch or plastic material. Some authors, however, have recommended a partial resection of the tumor, without recurrence ${ }^{4}$.

The follow-up of asymptomatic patients that were not submitted to surgery must include anticoagulation therapy, although its effectiveness in protecting against embolic phenomena is controversial. The conduct when an isolated lesion is identified on the right side includes the surgical removal, when the mass is large and/or mobile, or in the presence of patent foramen ovale, due to the possibility of paradoxical embolism.

The PFE courses favorably with the surgical resection and one can even refer to cure, although it is recommended to perform an annual echocardiographic follow-up for the possible detection of rare recurrence cases ${ }^{4}$.

To date, no imaging technique is capable of establishing a definitive diagnosis of these tumors. Only endomyocardial biopsies or thoracotomy are capable of characterizing in vivo such cardiac masses. The prompt histological analysis is crucial to confirm the diagnosis and establish the most adequate surgical treatment.

\section{Potential Conflict of Interest}

No potential conflict of interest relevant to this article was reported.

\section{Sources of Funding}

There were no external funding sources for this study.

\section{Study Association}

This study is not associated with any post-graduation program. 


\section{References}

1. Grande AM, Ragni T, Vigano M. Primary cardiac tumors: a clinical experience of 12 years. Tex Heart Inst J. 1993; 20: 223-30.

2. Caballero J, Calle G, Arana R, Sancho M, Caballero FJ, Piñero C, et al. Cardiac papillary fibroelastoma: different forms of the clinical presentation. Rev Esp Cardiol. 1997; 50 (11): 815-7.

3. Oliveira SF, Dias RR, Fernandes F, Stolf NA, Mady C, Oliveira SA. Cardiac papillary fibroelastoma: experience of an institution. Arq Bras Cardiol. 2005; 85 (3): 205-7.

4. Dein JR, Frist WH, Stinson EB, Miller DC, Baldwin JC, Oyer PE, et al. Primary cardiac neoplasms: early and late results of surgical treatment in 42 patients. J Thorac Cardiovasc Surg. 1987; 93 (4): 502-11.

5. Limandri G, Homma S, Di Tullio MR, Hodges D, Arora R, Marboe C, et al. Detection of multiple papillary fibroelastomas of the tricuspid valve by transesophageal echocardiography. J Am Soc Echocardiogr. 1994; 7 (3 Pt 1): 315-7.

6. Salcedo EE, Cohen GL, Write RD, Davison MB. Cardiac tumors: diagnosis and treatment. Curr Probl Cardiol. 1992; 17: 73-137.

7. Lane GE, Kapples EJ, Thompson RC, Grinton SF, Finck SJ. Quiescent left atria myxoma. Am Heart J. 1994; 127 (6): 1629-31.

8. Fujita N, Caputo GR, Higgins CB. Diagnosis and characterization of intracardiac masses by magnetic resonance imaging. Am J Card Imaging. 1994; 8 (1): 69-80.

9. Fueredi GA, Knetchtges TE, Czarnecki DJ. Coronary angiography in atrial myxoma: Findings in nine cases. AJR. 1998; 152: 737-8. 